\title{
Comparison of Geriatric Assessment Tools in Patients with Solid Malignancies
}

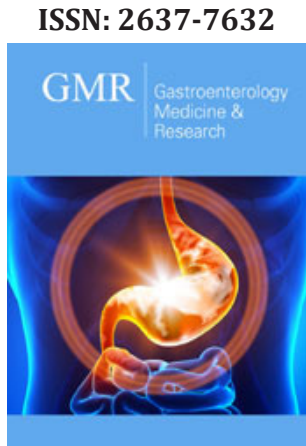

*Corresponding author: Antoine Abi Abboud, Department of Gastroenterology, Lebanese University Faculty of Medical Sciences, Lebanon

Submission: 眥 September 16, 2019

Published: 战 October 03, 2019

Volume 3 - Issue 4

How to cite this article: Nancy N Hajjar, Maroun H Eid, Antoine Abi Abboud, Evelyne G Helou. Comparison of Geriatric Assessment Tools in Patients with Solid Malignancies. Gastro Med Res. 3(4). GMR.000570. 2019.

DOI: 10.31031/GMR.2019.03.000570

Copyright@ Antoine Abi Abboud, This article is distributed under the terms of the Creative Commons Attribution 4.0 International License, which permits unrestricted use and redistribution provided that the original author and source are credited.

\author{
Nancy N Hajjar ${ }^{1}$, Maroun H Eid ${ }^{2}$, Antoine Abi Abboud ${ }^{3 *}$ and Evelyne G Helou ${ }^{4}$ \\ ${ }^{1}$ Department of Dermatology, Lebanese University Faculty of Medical Sciences, Lebanon \\ ${ }^{2}$ Department of Ophtalmology, Lebanese University Faculty of Medical Sciences, Lebanon \\ ${ }^{3}$ Department of Gastroenterology, Lebanese University Faculty of Medical Sciences, Lebanon \\ ${ }^{4}$ Department of Hematology-Oncology, Lebanese University Faculty of Medical Sciences, \\ Lebanon
}

\begin{abstract}
Background: Eastern Cooperative Oncology Group Performance Status (ECOG PS), and Karnofsky Performance Status (KPS) are well known screening tools for older cancer patients. However, the Comprehensive Geriatric Assessment score (CGA), which is a multidimensional evaluation of the older patient, is less used in practice. Few comparative studies were done before and there is no screening tool uniformly used worldwide.
\end{abstract}

Methods: One hundred patients aged 65 years and older diagnosed with solid tumors were interviewed before starting their first chemotherapy cycle. The scores of ECOG PS, KPS and CGA were filled. The statistical analysis was done using the Chi-square test.

Results: No correlation was found between CGA fragile on one side and ECOG $\geq 2$ / KPS $\leq 70 \%$ on the other side with a p-value of 0.9 and 0.48 respectively. Forty three per cent of patients had a good performance status according to ECOG PS and KPS despite a fragile CGA.

Conclusion: KPS and ECOG PS may not be reliable for treatment decision making in the older oncology patient.

Keywords: Cancer; ECOG PS; KPS; CGA; Chemotherapy

Abbreviations: CGA: Comprehensive Geriatric Assessment; ECOG PS: Eastern Cooperative Oncology Group Performance Status; KPS: Karnofsky Performance Status

\section{Introduction}

Aging affects significantly the incidence of cancer and its mortality rate [1]. Thus, oncologists spend significant time and effort caring for elderly patients (defined as aged 65 years and older) in their everyday practice [2]. Despite the high incidence of cancer in this group, older patients have been under-represented in clinical trials that set the standards for care in oncology practice [2]. Only scarce data exist regarding the risks and benefits of cancer treatment in this population, and there are not enough guidelines that specifically address the evaluation and treatment of the older cancer patient [2]. Evidence suggests that chemotherapy toxicities are influenced by the patient's comorbidities, cognitive impairment and limited social support [3]. This is due to the age-related physiologic changes seen at the cellular level that are responsible for the vulnerability of elderly patients [3]. Several oncological screening tools exist. The aim of these scores is to detect if the patients are "fit" to receive chemotherapeutic agents [2]. The CGA consists of a multidimensional evaluation of the general health status but also integrates functional, cognitive, social and psychological parameters [4]. The commonly used performance status scores (KPS or ECOG PS scales) take into consideration few criteria in the assessment of the elderly cancer patient and thus may miss areas of vulnerability that are more frequently found in this older category of patients $[5,6]$. The KPS score was initially founded in 1948, it consists of a scale from $0 \%$ to $100 \%$ with $10 \%$ difference between every scale where $100 \%$ is normal and $0 \%$ is death [6].

Concerning the ECOG PS score, it was initially introduced in 1982 as five categories scale where 0 is being asymptomatic and 5 is death which makes it a more simple scale comparing to KPS [6]. Few studies have been conducted to assess the correlation between the CGA on one side and the KPS and ECOG on the other side. Screening older cancer patients is recommended 
by both the National Comprehensive Cancer Network (NCCN) and the International Society for Geriatric Oncology (ISGO) without a defined screening tool $[7,8]$. However, although a CGA score may guide physicians through treatment decisions, it may not be practical for everyday practice considering its length (45 minutes interview) [2]. Therefore, identification of the most beneficial score of patient assessment is important.

\section{Methods}

\section{Patients}

We enrolled 100 patients aged 65 years and older diagnosed with solid malignancies who were coming to receive their first chemotherapy cycle in three tertiary care centers from December 2015 to January 2018.

Patients who satisfied the inclusion criteria signed an informed consent.

\section{Categorization}

We adapted the clinical interview method to fulfill the ECOG PS score, the KPS as well as the CGA, before initiation of any sort of treatment. Patients were divided into three categories for each score:

1. ECOG PS: $0-1-\geq 2$.

2. KPS: $90-100 \%-80 \%-\leq 70 \%$.

3. CGA: Normal- Vulnerable- Fragile.

Two tables were drawn, one comparing the CGA to the ECOG PS, and the other comparing the CGA to the KPS, in order to determine the number of patients in each zone and most importantly the ones in the danger zone. These patients were considered medium or low risk patients according to the ECOG PS or the KPS, but fragile or high risk patients according to the full CGA score.

\section{CGA}

Instrumentations used in the CGA were the following: Activities of Daily living (ADL), Instrumental Activities of Daily living, the Fall Risk Assessment, the Pain scale, the Fatigue scale, Mini Mental Status Exam (MMSE), Comorbidities, Mini Nutritional Assessment (MNA), and Hospital Anxiety and Depression Scale (HADS). Cut-off levels for classifying patients as normal, vulnerable or fragile in the CGA score are not yet validated. In our study, we used the Balducci classification.

\section{Statistics}

All the statistical analysis was done using the SAS software and Chi-square test. The level of $\mathrm{p}<0.05$ was considered as significant.

\section{Result}

\section{Patients' characteristics}

For a total of 100 patients: The median age was 72 with a range between 65 and 88 . Fifty five per cent were women and $45 \%$ were men. Sixty five percent were smokers or ex- smokers and 35\% were non- smokers. In addition, 70.7\% of smokers had a fragile CGA ( $p$ $<0.006)$. The most common organ involved was the lung (21\%), the second was the breast (20\%) and the third was the colon (18\%) other localizations included: Uterus (9\%), Bladder (8\%), Pancreas $(8 \%)$, Stomach (7\%), Ovaries (5\%), and Cervix (4\%). The results are shown in Figure 1.

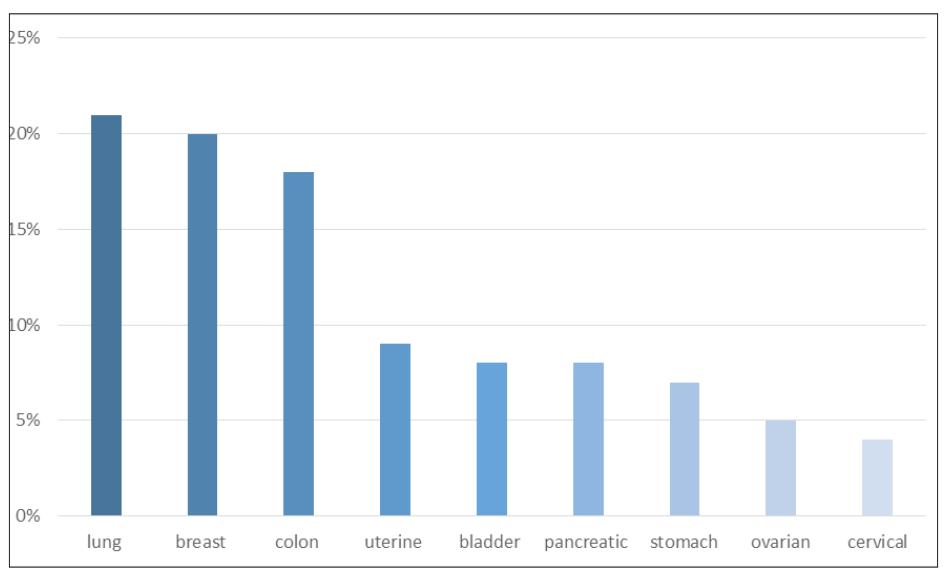

Figure 1: Percentage of the organs involved.

\section{Scores}

The median score of ECOG PS was 1: 26\% ECOG PS $=0,45 \%$ ECOG PS $=1,29 \%$ ECOG PS $\geq 2$. The median score of KPS was 80: 39\% KPS 90-100\%, 34\% KPS $80 \%$, 27\% KPS $\leq 70 \%$. Most of patients had a fragile CGA: $19 \%$ normal, $20 \%$ vulnerable, and $61 \%$ fragile. Each category of CGA was analyzed alone according to the organ involved in order to determine the impact of the organ on the performance status of the patient if present. Only in the category of breast cancer, all the three classes of CGA were in close repartition. Results are shown in Figure 2. The comparison of scores showed that $43 \%$ of patients were considered as fragile according to the CGA but with a good performance status according to ECOG PS or KPS thus defined as being in a danger zone. Results are shown in Figure 3. In order to assess whether there is a correlation or not 
between the KPS, ECOG PS and CGA scores, we run a correlation test using the Chi-square test. No correlation was found between the results of CGA on one side and KPS or ECOG PS on the other side with $p$ values $>0.05$. However, the results of ECOG PS and KPS were correlated with a $\mathrm{p}<0.0001$. Results are shown in Table 1 .

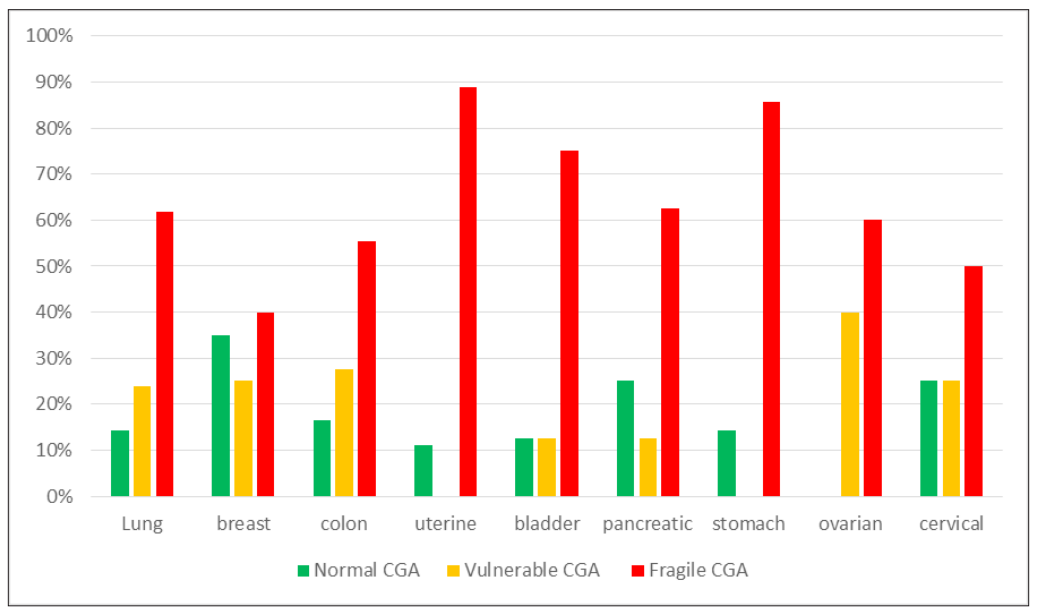

Figure 2: CGA categories according to each organ.

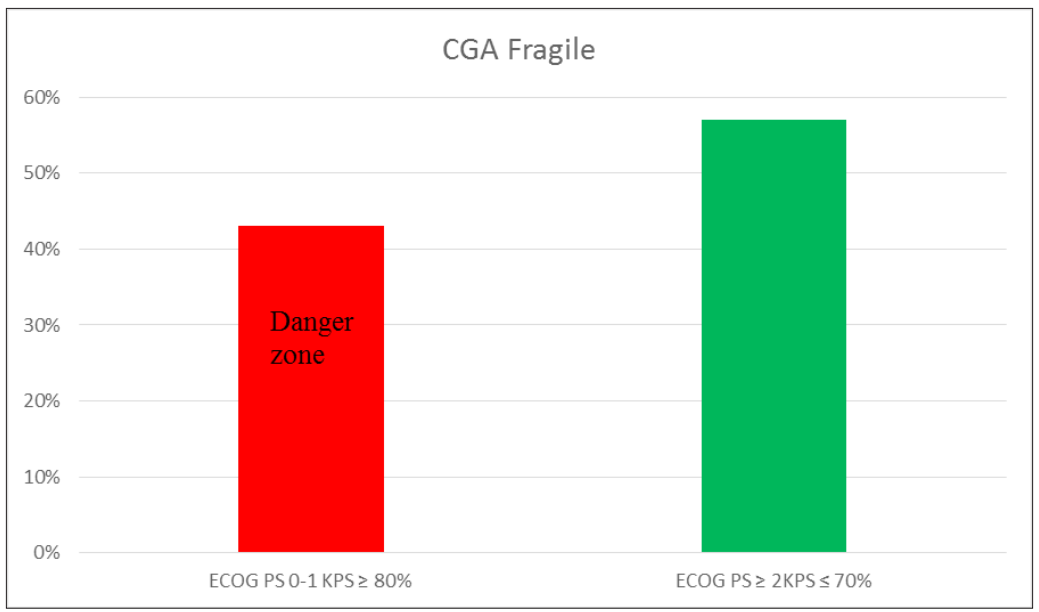

Figure 3: ECOG PS and KPS scores in the CGA fragile category.

Table 1: Results of ECOG PS/ KPS and CGA comparison.

\begin{tabular}{|c|c|c|c|c|}
\hline & $\begin{array}{c}\text { ECOG PS }=0 \\
\text { KPS } \mathbf{9 0 - 1 0 0 \%}\end{array}$ & $\begin{array}{c}\text { ECOG PS }=1 \\
\text { KPS }=\mathbf{8 0} \%\end{array}$ & $\begin{array}{c}\text { ECOG PS } \geq 2 \\
\text { KPS } \leq 70 \%\end{array}$ & p-value \\
\hline \multirow{2}{*}{ CGA Normal } & $\mathrm{N}=11$ & $\mathrm{~N}=8$ & $\mathrm{~N}=0$ & $\mathrm{P} 0.7198$ \\
& $\mathrm{~N}=14$ & $\mathrm{~N}=4$ & $\mathrm{~N}=0$ & $\mathrm{P} 0.7549$ \\
\hline \multirow{2}{*}{ CGA Vulnerable } & $\mathrm{N}=4$ & $\mathrm{~N}=5$ & $\mathrm{~N}=11$ & $\mathrm{P} 0.2131$ \\
& $\mathrm{~N}=5$ & $\mathrm{~N}=6$ & $\mathrm{~N}=10$ & $\mathrm{P} 0.3580$ \\
\hline \multirow{2}{*}{ CGA Fragile } & $\mathrm{N}=11$ & $\mathrm{~N}=32$ & $\mathrm{~N}=18$ & $\mathrm{P} 0.9070$ \\
& $\mathrm{~N}=19$ & $\mathrm{~N}=24$ & $\mathrm{~N}=18$ & $\mathrm{P} 0.4848$ \\
\hline
\end{tabular}

\section{Discussion}

In our study, ECOG PS and KPS scores were correlated with both detecting the same number of patients in the danger zone. Thus, one can think of using either of these tests with no superiority in terms of detecting vulnerable patients. Several studies showed a close agreement in these two scores. For instance, a study conducted by Hollen PJ [9] and colleagues, demonstrated the presence of high correlation between ECOG PS and KPS in 75 patients with advanced non-small cell lung cancer prior to initiation of chemotherapy with a Pearson's ratio of 0.75 [9]. In other studies, the KPS score was inferior to ECOG PS in terms of predicting chemotherapy toxicities. For example, a study conducted by Buccheri G and collaborators compared these two scales in 536 patients with lung cancer before and after receiving chemotherapy. The results showed a better 
predictive capacity of ECOG PS in clinical decision making [10]. As discussed earlier, although the CGA covers an important number of domains not included in other geriatric assessment tools, is time consuming for the examiner and it requires patient cooperability and examiner expertise [2].

However, $43 \%$ of patients included in this study were found in the danger zone defined as having an ECOG PS $0-1$ or KPS $\geq 80 \%$, in other terms having a good performance status, along with a fragile CGA. In fact, 43 patients who should not receive chemotherapy according to the CGA score were considered suitable candidates for chemotherapy based on the ECOG PS and KPS scores and have undergone treatment. Similar results were found in a study conducted by Owusu C and colleagues, where 117 patients aged $\geq 65$ years old with newly diagnosed cancer were assessed. In $43 \%$ of patients, two or more geriatric abnormalities in CGA evaluation not detected with ECOG PS or KPS were found. Thus, suggesting a full geriatric evaluation in older oncology patients in order to ameliorate cancer outcome in this population [11]. Looking at the other results of this study, we noted that no patients fell in the category of both an ECOG PS score of $\geq 2$ or KPS $\leq 70 \%$ along with a normal CGA. Thus, the ECOG PS and KPS scores could be used as surrogate for the CGA when ECOG PS score is $\geq 2$ and/or KPS score is $\leq 70 \%$; these two categories of patients would simply be considered ineligible for chemotherapy without the need to do a full CGA. Conversely, patients with an ECOG PS score of 0-1 and/ or KPS score of $\geq 80 \%$ would need to undergo a full CGA for better assessment of whether or not, they are candidates for standard chemotherapy regimens. Suggested algorithm is shown in Figure 4.

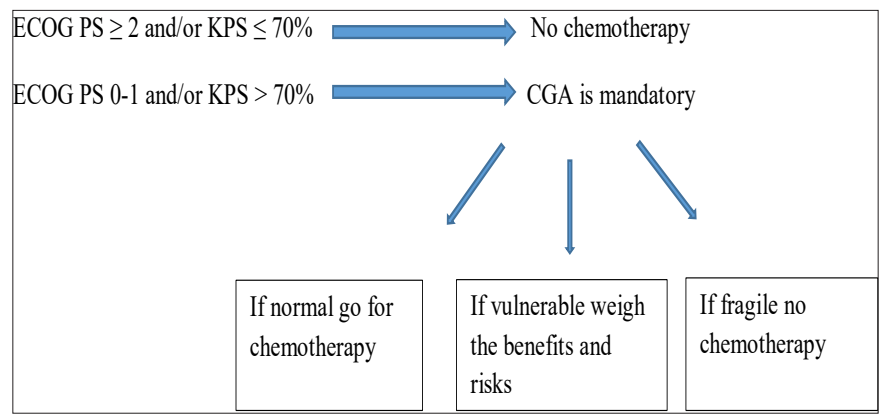

Figure 1: Suggested algorithm for treatment decision making.

In our study, $65 \%$ of patients were smokers or ex-smokers. The results showed a strong correlation between smoking status on the one hand and ECOG score of $\geq 2, \mathrm{KPS} \leq 70 \%$ and fragile overall CGA on the other hand $(\mathrm{p}<0.006)$. Thus witnessing the detrimental effect of smoking on the patient general health status and physiologic reserve. In the review of literature, studies that evaluate the impact of smoking on performance status were not found. Moreover, no published data on the relation between cigarettes' consumption and chemotherapy toxicities were found. The limitations of this study are mainly the lack of follow-up to register Overall Survival (OS) and Progression Free Survival (PFS) in order to determine the consequences of chemotherapy administration in the danger zone, as well as the lack of clear cut-off levels for classifying patients as normal, vulnerable or fragile in the CGA.

\section{References}

1. Jemal A, Siegel R, Xu J (2010) Cancer statistics. CA Cancer J Clin 60(5): 277-300.

2. Hutchins LF, Unger JM, Crowley JJ (1999) Underrepresentation of patients 65 years of age or older in cancer treatment trials. N Engl J Med 341(27): 2061-2067.

3. Dale W, Mohile SG, Eldadah BA (2012) Biological, clinical, and psychosocial correlates at the interface of cancer and aging research. J Natl Cancer Inst 104(8): 581-589.

4. Yee KW, Pater JL, Pho L (2003) Enrollment of older patients in cancer treatment trials in Canada: Why is age a barrier? J Clin Oncol 21(8): 1618-1623.
5. Trimble EL, Carter CL, Cain D (1994) Representation of older patients in cancer treatment trials. Cancer 74(7): 2208-2214.

6. Puts MT, Santos B, Hardt J (2014) An update on a systematic review of the use of geriatric assessment for older adults in oncology. Ann Oncol 25(2): 307-315.

7. Extermann M, Aapro M, Bernabei R (2005) Use of comprehensive geriatric assessment in older cancer patients: Recommendations from the task force on CGA of the International Society of Geriatric Oncology (SIOG). Crit Rev Oncol Hematol 55(3): 241-252.

8. Decoster L, Van Puyvelde K, Mohile S (2015) Screening tools for multidimensional health problems warranting a geriatric assessment in older cancer patients: An update on SIOG recommendations. Ann Oncol 26(2): 288-300.

9. Hollen PJ, Gralla RJ, Stewart JA (2005) A prospective comparison of Karnofsky (KPS) with ECOG performance status in patients with nonsmall cell lung cancer (NSCLC): A COMET group study investigating sensitivity and specificity issues important in clinical decision making. J Clin Oncol 23(16): 8134-8134.

10. Buccheri G, Ferrigno D, Tamburini M (1996) Karnofsky and ECOG performance status scoring in lung cancer: A prospective, longitudinal study of 536 patients from a single institution. Eur J Cancer 32(7): 11351141.

11. Owusu C, Koroukian S, Schluchter M (2011) Screening older cancer patients for a comprehensive geriatric assessment: A comparison of three instruments. J Geriatr Oncol 2(2): 121-129.

For possible submissions Click below: 